\title{
Removing Camera Shake using Discrete Cosine Transform
}

\author{
D. Ramesh Varma \\ Assistant Professor, ECE \\ Shri Vishnu Engineering \\ College for Women
}

\author{
D. Krishna Madhav \\ Assistant Professor, ECE \\ Shri Vishnu Engineering \\ College for Women
}

\author{
K. Rajasekhar \\ Assistant Professor, ECE \\ Shri Vishnu Engineering \\ College for Women
}

\begin{abstract}
Image restoration is one of the burning issues in the field of image processing. Generally, images are corrupted or damaged due to the noise present in the system or due to motion blur while capturing the image. In this paper, a problem of removing blurness in an image which is caused due to camera shake is discussed. The blur Kernel in an image is uneven. Because of this reason, every image in a burst of images is blurred in a different way. In this paper, a new technique is proposed in which burst of images are taken and calculates a weighted average in discrete cosine domain, where the weights depend on their discrete cosine spectrum magnitudes.
\end{abstract}

\section{Keywords}

Discrete cosine spectrum, motion blur, camera shake.

\section{INTRODUCTION}

Taking images is one of the challenging experiences in the field photography. Accumulation of photons in the sensor is the basic principle of photography in a given exposure time. The quality of image increases when more photons reach the surface of the sensor. The blurriness in an image occurs due to accumulation of photons in the neighbouring pixels.

The mathematical model of camera shake is modelled as

$$
y=x * b+n
$$

where $y$ is the blurred image

$$
\begin{aligned}
& \mathrm{b} \text { is the blur kernel } \\
& \mathrm{n} \text { is additive noise }
\end{aligned}
$$

The blurring kernel is obtaining by relative motion between the camera and object (scene).Due the advancement in the technology, various applications has been created. There is a burst mode in the camera by which we could take burst of images at a single shot when capturing the picture, the blueness occurs due to camera shake or relative motion of the scene. Therefore, in each image of the burst, camera moves independently and differently for different images in a burst. Therefore the blur occurred is different from one in another image of the burst. In this paper, a new algorithm is presented in which burst of blurred images are taken as input and then computes weighted average of the discrete cosine coefficients of the images from the burst of images. The paper is organized as follows. Section I gives the introduction, Section II gives the literature review, Section III gives explains the amassing(accumulation) of burst of images in discrete cosine domain to reconstruct an image, Section IV elaborates the implementation of an algorithm and flow chart, Section V gives the experimental results and section VI gives the performance metrics followed by conclusion and references.

\section{LITERATURE REVIEW}

There are various deblurring algorithms. They are classified into two groups.

1. single image based algorithms

2. multiple image based algorithms

\subsection{Single image based algorithms}

If the blur kernel is shift invariant, the problem of restoring the image from the single blurred image can be done using image deconvolution [1]. The image deconvolution process is done in the two ways. one is by known kernel and other is by unknown kernel. In the second case kernel is not known so, first the kernel is estimated and then the image is recovered .In $[1,2,3,6]$ the authors concluded that it is better to first solve a maximum a posterior estimation of the kernel than of the latent image and kernel simultaneously.

\subsection{Multiple image based algorithms}

In this method, blurring kernels are estimated using two or more images. So, there are different exposure timings for two images. Therefore the problem for this method is that exposure timings are different for two images. Short exposure image is a sharp image with noise content where as long exposure image is a blurred one with less noise by using sharper one, motion kernel is estimated [2].

\subsection{FFT based removing blurness in an Image [5]}

Fast fourier transform is having truncation problem and also have poor energy compaction. It cannot extract enough frequencies without enough samples. The implicit periodicity of DFT gives rise to boundary discontinuous that results in significant high frequency content.

\section{DISCRETE COSINE BURST AMASSING}

Generally, the camera shake generated due to handshake is random $[3,4]$. The independent movement of the hand causes the shake of the camera. Due to this, the blurness occurs in an image. In this, a burst of $\mathrm{N}$ images of same scene are taken.

$$
G_{i}=k_{i}+\eta_{i} ; \text { For } i=1,2 \ldots \ldots \ldots \ldots
$$

In this kernels are different for different images. Due to this, discrete cosine transform of each image of the burst is different. In the proposed method, the reconstruction is done for an image which is having largest value of discrete cosine magnitude in the burst.

\subsection{Discrete Cosine Magnitude Weights}

Weights of images can be calculated as;

$$
w_{i}(\zeta)=\frac{\left|\widehat{G_{l}(\zeta)}\right|^{Q}}{\sum_{j=1}^{N}\left|\overline{G_{l}(\zeta)}\right|^{Q}}
$$




$$
R_{Q}(x)=E^{-1}\left(\sum_{i=1}^{N} w_{i}(\zeta) \cdot \hat{G}_{i}(\zeta)\right)(x) .
$$

Where $\hat{G}_{i}$ represents the DCT of burst images. $R_{Q}$ is the reconstructed image. E denotes the discrete cosine transform.

\subsection{Equivalent PSF}

The reconstructed image $R_{Q}$ can be obtained by the convolution between average kernel and sharp image.

$$
R_{Q}=R * k_{D C}+\bar{\eta}
$$

Where $k_{D C}(x)=E^{-1}\left(\sum_{i=1}^{N} w_{i}(\zeta) \cdot \hat{k}_{i}(\zeta)\right)(x)$

$\bar{\eta}$ is the weighted average of the noise

$k_{D C}$ is constructed from least attenuated frequencies in the burst given by the weights of images. Convolution kernels may introduce phase distortion in the images [6,7]. Therefore, $k_{D C}$ may not be closer to dirac function. Blurring kernels are estimated using

$$
\left\|R_{\text {ref }} * k_{i}-G_{i}\right\|
$$

Where $R_{\text {ref }}$ is the sharp reference image [7]. Kernels are estimated using reference image by minimizing the least squares distance to the blurred acquisition.

\section{ALGORITHM, FLOWCHART OF PROPOSED METHOD}

\section{Algorithm Implementation}

Step1: Take a burst of images [8] .Among them, register less blurred image.$G_{i}$ where $i=1,2, \ldots . N$

Step2: Calculate the discrete cosine transform for the registered images $\hat{G}_{i}$ Where $\mathrm{i}=1, \ldots . \mathrm{N}$

The kernel does not vary spatially because of the camera shake

Step3: $\left|\widehat{G}_{i}\right|$ is a low pass filtered before computing weights.

$$
\left|\hat{G}_{i}\right|=\mathrm{F}_{\sigma}\left|\hat{G}_{i}\right|
$$

Where $F_{\sigma}$ is a gaussian filter of standard deviation $\sigma$. The strength of the LPF depends on the assumed motion kernel size.

Step4: Set $\sigma=\min \left(\mathrm{m}_{\mathrm{h}}, \mathrm{m}_{\mathrm{w}}\right) / \mathrm{K}_{\mathrm{s}}$ Where $\mathrm{K}_{\mathrm{s}=} 50$ pixels where $m_{h} \times m_{w}$ is the size of image.

Step5: The final discrete cosine burst amassing is given as

$$
\begin{aligned}
& \qquad R_{Q}=E^{-1}\left(\sum_{i=1}^{N} w_{i} \cdot \hat{G}_{i}\right) \\
& \text { Where } w_{i}=\left|\bar{G}_{i}^{\wedge}\right|^{Q} / \sum_{j=1}^{N}\left|\bar{G}_{i}^{\wedge}\right|^{Q}
\end{aligned}
$$

Step6: For better visualization of reconstructed image, apply image sharpening using unsharp masking filter.

\section{Flow chart}

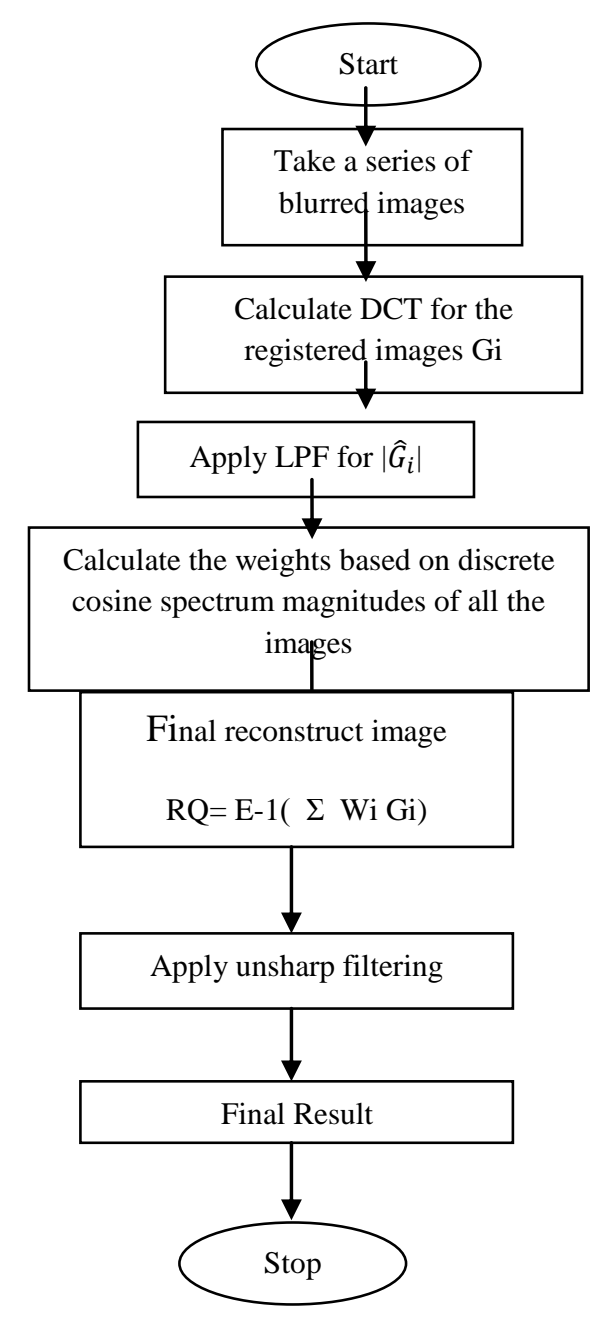

\section{EXPERIMENTAL RESULTS}

In this paper, a group of images are taken and weighted average filter using DCT is applied. The results were compared with the other methods like weighted average filter using DFT and DWT [9].
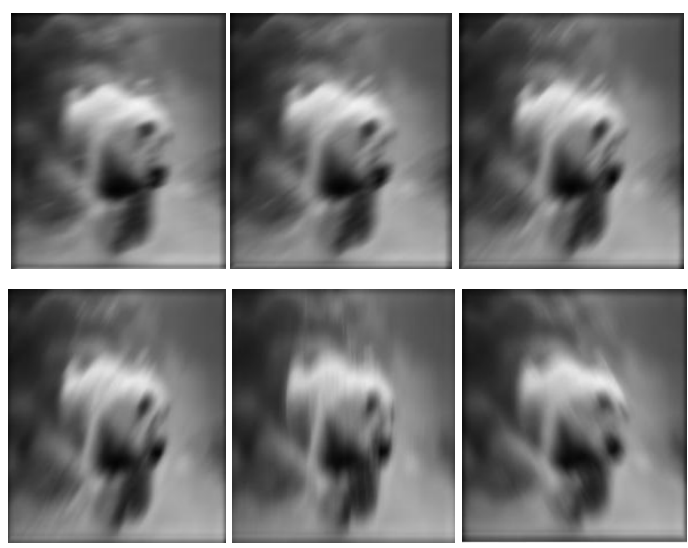

Fig.1. Group of Blurred Images 


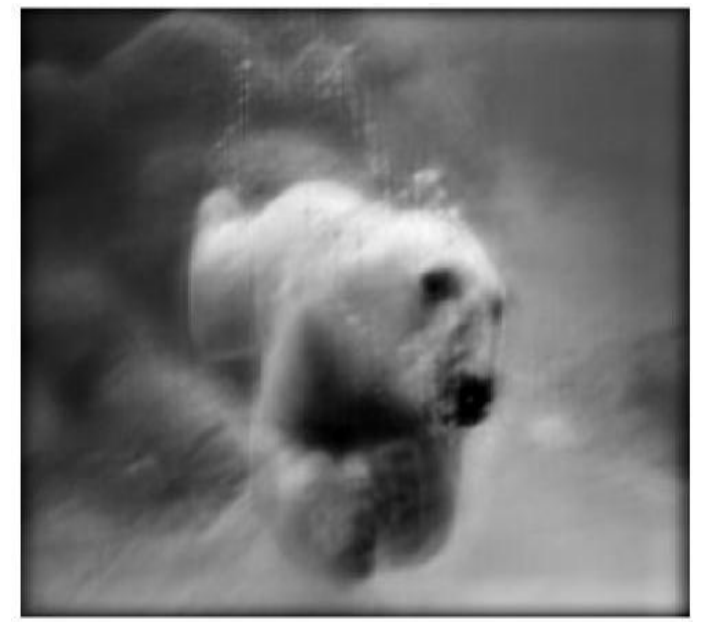

Fig.2. Reconstructed image using DCT

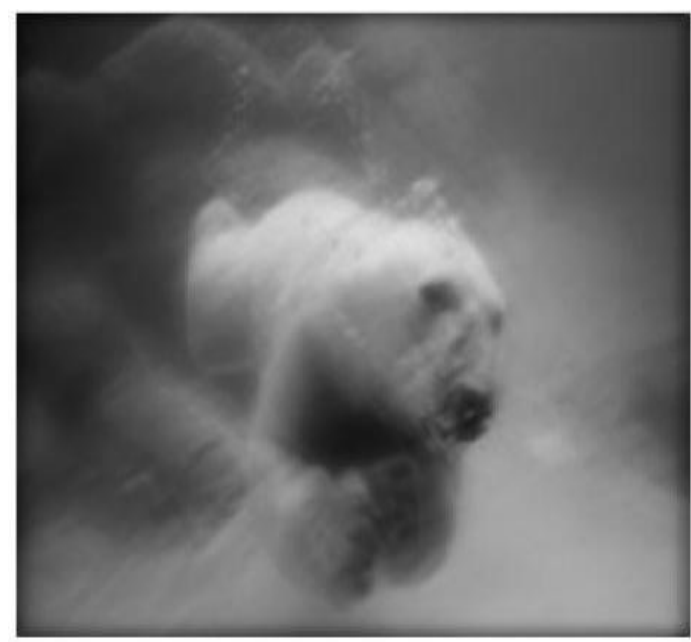

Fig.3.Reconstructed image using FFT

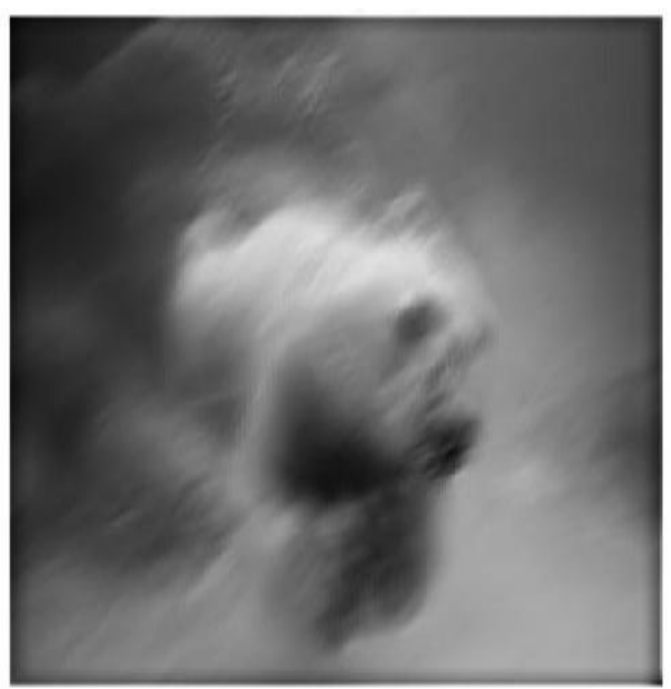

Fig.4. Reconstructed image using DWT

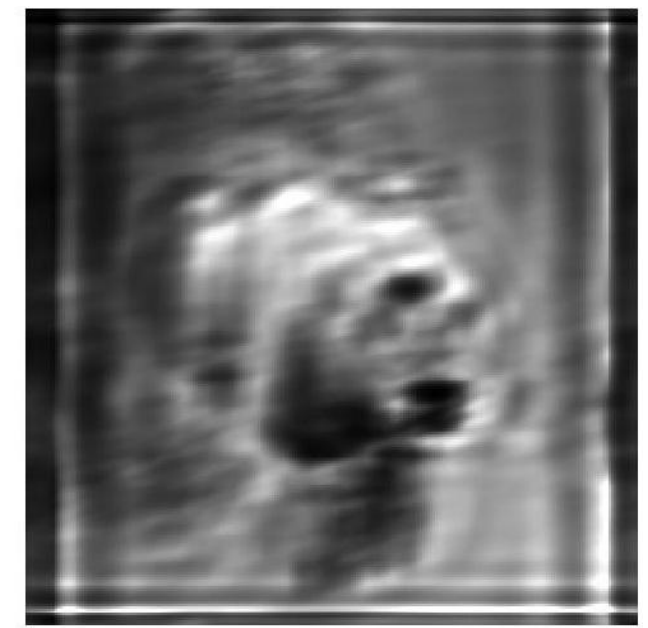

Fig.5. Reconstructed image using Blind deconvolution

\section{PERFORMANCE METRICS}

\begin{tabular}{|c|c|c|c|}
\hline S.No & Image & Method & PSNR \\
\hline \multirow{4}{*}{1.} & \multirow{4}{*}{ Underwater } & FFT & 32.60 \\
\hline & & DWT & 34.19 \\
\hline & & BLIND & 26.07 \\
\hline & & DCT(Proposed) & 34.21 \\
\hline \multirow{4}{*}{2.} & \multirow{4}{*}{ Moon surface } & FFT & 31.02 \\
\hline & & DWT & 32.89 \\
\hline & & BLIND & 25.95 \\
\hline & & DCT(Proposed) & 32.93 \\
\hline \multirow{4}{*}{3.} & \multirow{4}{*}{ Rice } & FFT & 26.35 \\
\hline & & DWT & 27.75 \\
\hline & & BLIND & 22.03 \\
\hline & & DCT(Proposed) & 27.79 \\
\hline
\end{tabular}

\section{CONCLUSION}

In this paper, camera shake is removed with the help of discrete cosine transform without calculating the blurring kernel. The reconstruction of an original image is done from the group of its blurred images. In this technique, discrete cosine transform is used for retrieving the original image. The DCT coefficients are having the ability to represent the regularity among the pixels [10]. Discrete cosine transform is having higher energy compaction when compared to DFT and DWT. This technique gives sharper and less noisy images when compared to state of art techniques. From the performance metrics, DWT and DCT are having approximately same PSNR value. But, when taking the visualization into consideration, DCT is better when compared to DWT.

\section{FUTURE SCOPE}

The future scope of this work is that the camera shake can be removed using 3-dimmensional transform which give good results because $3 \mathrm{D}$ platform is much preferable than the $2 \mathrm{D}$ platform. 


\section{REFERENCES}

[1] Q. Shan, J. Jia, and A. Agarwala, "High-quality motion deblurring from a single image," ACM Trans. Graph., vol. 27, no. 3, 2008, Art. ID 73.

[2] J. F.Cai,H.Ji, C.Liu, and Z.Shen,"Blind motion deblurring using multiple images," J.Comput.Phys., vol. 228, no. 14, pp.5057-5071,2009.

[3] F. Gavant, L. Alacoque, A. Dupret,and D. David, "A physiological camera shake model for image stabilization systems," in Proc. IEEE Sensors.

[4] F. Xiao, A.Silverstein, and J. Farrell, "Camera-motion and effective spatial resolution," in Proc. Int. Congr.Imag. Sci. (ICIS), 2006,pp. 33-36.

[5] Removing Camera Shake via Weighted Fourier Burst Accumulation by Mauricio Delbracio and Guillermo Sapiro.
[6] X..Zhu, F.Šroubek, and P. Milanfar, "Deconvolving PSFs for a better motion deblurring using multiple images" in Proc.IEEE12th Eur.Conf.Comput.Vis. (ECCV), Oct. 2012, pp. 636-647.

[7] M.Delbracio, P.Musé, A.Almansa, and J.-M. Morel, "The non-parametric sub-pixel local point spread function estimation is a well posed problem," Int. J. Comput. Vis. vol. 96, no. 2, pp. 175-194, 2012.

[8] B. Zitová and J. Flusser, "Image registration methods: A survey," Image Vis. Comput., vol. 21, no. 11, pp. $977-$ 1000,2003

[9] "A New Weighted Average Filter for Removing Camera Shake" International Journal of Computer Applications (0975 - 8887) Volume 156 - No 9, December 2016

[10] "Image Restoration using 3-Dimensional Discrete Cosine Transform" International Journal of Computer Applications (0975 - 8887) Volume 156 - No 9, December 2016. 